TITLE:

\title{
Numerical investigations of the dynamics of two-component vesicles.
}

\author{
$\operatorname{AUTHOR}(\mathrm{S})$ : \\ Taniguchi, Takashi; Yanagisawa, Miho; Imai, \\ Masayuki
}

\section{CITATION:}

Taniguchi, Takashi ... [et al]. Numerical investigations of the dynamics of two-component vesicles.. Journal of physics. Condensed matter 2011, 23(28): 284103.

\section{ISSUE DATE:}

2011-07-20

URL:

http://hdl.handle.net/2433/144629

\section{RIGHT:}

(C) IOP Publishing 2011.; This is not the published version. Please cite only the published version.; この論文は出版社版でありません。引用の 際には出版社版をご確認ご利用ください。 


\title{
Numerical investigations of the dynamics of two-component vesicles
}

\author{
Takashi Taniguchi ${ }^{1,2}$, Miho Yanagisawa ${ }^{3}$, Masayuki Imai ${ }^{4}$ \\ 1 Department of Chemical Engineering, Kyoto University, Kyoto 606-8510, Japan \\ 2 CREST/JST, Kawaguchi, Saitama, 332-0012, Japan \\ 3 Department of Physics, Kyoto University, Sakyo, Kyoto 606-8502, Japan and \\ 4 Department of Physics, Ochanomizu University, Otsuka, Bunkyo, Tokyo 112-8610, Japan
}

\begin{abstract}
We examined the dynamics of the deformation and phase separation of two-component vesicles. First, we numerically investigated the effects of (i) thermal noise, (ii) hydrodynamic flow induced by the line tension of the domain boundary and (iii) composition-dependent bending rigidity on the coarsening dynamics of a phase-separated pattern on the surfaces of vesicles with fixed shapes. The dynamical exponent $z\left(N_{\mathrm{DB}} \sim t^{-z}\right.$, the total length of the domain boundaries) of the coarsening of phase-separated pattern was found to decrease from $z=1 / 3$ under no thermal noise to $1 / 5<z<1 / 4$ when including the effects of thermal noise. We also found that the hydrodynamic effect enhances the coarsening in a bicontinuous phase separation for a spherical vesicle. In phase separations of a shape-fixed tubular vesicle, a band-like phase separation with periodicity along the longer axis of the tube occurs because of the composition-dependent bending rigidity and the higher curvatures at the tube end-caps. Second, we also explored the dynamics of shape deformation coupled with phase separation through the bending rigidity of the membrane which depends on the local composition in lipid, and found that the composition-dependent bending rigidity crucially influences the phase separation and deformation of the vesicle. The results of simulations are in good agreement with experimentally observed behaviors known as "shape convergence" [Yanagisawa et al, 2008 Phys. Rev. Lett. 100 148102].
\end{abstract}

PACS numbers: 64.75.St, 87.16.D-, 82.70.Uv

\section{INTRODUCTION}

It is well-known that lipid membranes and their closed forms, vesicles, exhibit a wide variety of shapes, including sphere, cup, biconcave, prolate dumbbell, pear, starfish, and that the transformations among these shapes occur through changes in environmental factors such as temperature, $\mathrm{pH}$ and osmotic pressure. These vesicle shape transformations have fascinated many scientists. Lipid bilayers seen in the membranes of biological cells, such as red blood cells, are in a fluid state at physiological temperature where lipid molecules can diffuse within the membranes. Therefore, such a membrane is referred to as a fluid membrane. Until the early 1960's, it was difficult to theoretically explain the shape deformations of fluid-membrane vesicles. After the pioneering works of Canham[1] and Helfrich[2], increasing numbers of researchers have theoretically and experimentally investigated the shape deformations of homogeneous fluid membrane vesicles caused by changes in environmental factors such as temperature, $\mathrm{pH}$ and osmotic pressure.

Recently, much attention has been paid to the membranes and vesicles of binary and/or ternary lipid mixtures [3-29] because these systems are a simpler model systems for understanding the behaviors of real biomembranes which are intrinsically composed of many kinds of lipids and membrane proteins. So far, shape deformations coupled with phase separation for multi-component vesicles, have been investigated theoretically[3-11] and experimentally[18-29]. Theoretically obtained equilibrium shapes of budding induced by the line tension of domain boundaries and/or local spontaneous curvature[3-
7] and phase diagrams describing shape deformations and phase-separated patterns in two-component vesicles $[10,11]$ have been reported. Dynamics of shape deformation and phase separation have been explored by numerical simulations using a generalized time-dependent Ginzburg-Landau equation coupled with shape deformation through a composition-dependent spontaneous curvature and domain boundary energy $[12,13]$, using Monte Carlo simulations $[14,15]$ and using dissipative particle dynamics (DPD) $[16,17]$. In experiments with vesicles composed of ternary lipid mixtures, Veatch and Keller have reported miscibility phase diagrams [19]. Critical phenomena in membranes of ternary lipid mixtures have also been investigated experimentally [21]. The critical behaviors of the membranes are reported to be consistent with those in the two-dimensional Ising universality class in comparisons between experimentally obtained and theoretically predicted critical exponents. Sakuma et al [27] have reported pore formation by temperature change in two-component vesicles composed of cone- and cylinder-like lipids. They also find that a rolled rim of the pore is induced by local spontaneous curvature at the pore periphery.

In the present paper, we focus on the dynamics of two-component vesicles composed of type-A and typeB lipids. In such membranes, if each type of lipid has an inherently different shape such as cylinder, cone, inversecone shapes and so forth, the local spontaneous curvature may depend on the local composition [3-13]. Furthermore, if a membrane composed only of each type of lipid exhibits different bending rigidity, the local bending moduli of a two-component membrane may depend 
on the local composition[3-8, 22-25]. In this context, Yanagisawa et al[23, 24] reported that the bending rigidity of a ternary component vesicle depends on its local composition, which crucially influences the dynamics of its phase separation and the vesicle deformation. In these experiments, the vesicle shapes are first controlled by an applied osmotic pressure difference at a higher temperature where the membrane exhibits a single phase. Next, the system temperatures are reduced to induce an intra-membrane phase separation. The results showed a wide variety of shape deformation dynamics coupled with phase separations. In addition, in the intramembrane phase separation, various shapes induced by osmotic pressure difference, e.g., prolate, oblate, tripod and starfish-like shapes, converge into oblate shapes consisting of two nearly flat circular domains rich in lipids with higher bending rigidity and a cylinder shaped domain rich in lipids with lower bending rigidity. This phenomenon is referred to as shape convergence.

The aim of the present work is to investigate the effect of intra-membrane heterogeneity in lipid composition on the shape deformation dynamics of two-component vesicles using a theoretical model that is an extension of the Canham-Helfrich bending elasticity model. Here we especially focus on the effects of (i) thermal noise, (ii) hydrodynamic flow induced by line tension at a domain boundary (i.e., hydrodynamic flow induced by a chemical potential gradient) and (iii) composition-dependent bending rigidity on the coarsening dynamics of the phase separation on fixed shape vesicles. Based on the understanding of the effects of (iii) on the phase separation dynamics of a fixed curved surface, we investigated the dynamics of shape deformation and phase separation from the view point of the coupling of the local composition and shape through the composition-dependent bending rigidity. Finally, we will clarify the origin of the shape convergence by investigating in detail the role of the composition-dependent bending rigidity in the time evolution of the systems where we prepared two types of initial shapes, i.e., (a) biconcave and (b) prolate dumbbell by controlling the osmotic pressure difference and changing the temperature to induce phase separations and shape deformations.

\section{MODEL OF A TWO-COMPONENT VESICLE}

We consider a two-component vesicle composed of a type-A and type-B lipids as a simpler model system of a real multi-component biomembrane. We treat the membrane as an infinitely thin sheet because the thickness of the membrane, $d$, is sufficiently small compared to the characteristic vesicle size, $R$, i.e., $(d \ll R)$. A position on the membrane specified by $u$ at time $t$ is expressed by $\boldsymbol{r}(u, t)$, where $u \equiv\left\{u^{1}, u^{2}\right\}$ parametrizes the membrane surface. The covariant vectors $\boldsymbol{g}_{\alpha}(\alpha \in\{1,2\})$ tangential to the surface can be obtained by taking a derivative with respect to $u^{\alpha}$ as $\boldsymbol{g}_{\alpha}=\partial \boldsymbol{r} / \partial u^{\alpha}$. A vector $\boldsymbol{n}$ normal to the

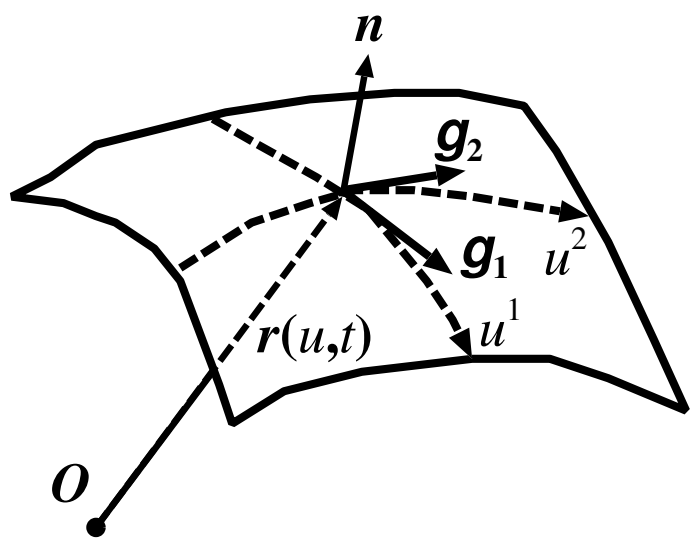

FIG. 1: A membrane portion treated as an infinitely thin sheet where the covariant tangential vector $\boldsymbol{g}_{\alpha}$ and unit vector $\boldsymbol{n}$ normal to the surface are drawn.

surface is given by $\boldsymbol{n}=\left(\boldsymbol{g}_{1} \times \boldsymbol{g}_{2}\right) / \sqrt{g}$, where $g$ is a metric defined by $g=\left(\boldsymbol{g}_{1} \times \boldsymbol{g}_{2}\right)^{2}$, and thus $\boldsymbol{n} \cdot \boldsymbol{n}=1$. The covariant metric tensor $g_{\alpha \beta}$ is defined using $\boldsymbol{g}_{\alpha}$ as $g_{\alpha \beta} \equiv \boldsymbol{g}_{\alpha} \cdot \boldsymbol{g}_{\beta}$. On the infinitely thin sheet, the composition fields can be defined. Assuming that the local compositions in the inner and outer layer of the bilayer membrane are identical, the fraction of the type-A and type-B lipids in a unit area at $u$ on the membrane are given by $\phi_{\mathrm{A}}(u, t)$ and $\phi_{\mathrm{B}}(u, t)$, respectively. We also assume that the membrane is laterally incompressible, which yields the following equation, $\phi_{\mathrm{A}}(u, t)+\phi_{\mathrm{B}}(u, t)=1$. Hence, the essential variables describing physical constants such as the bending modulus $\kappa$, the saddle splay modulus $\kappa_{\mathrm{G}}$, and the spontaneous curvature $H_{\mathrm{sp}}$ are functions of a local composition difference $\phi(u, t) \equiv \phi_{\mathrm{A}}(u, t)-\phi_{\mathrm{B}}(u, t)$. The total free energy functional, $F$, of the two-component vesicle with a total surface $S_{\mathrm{o}}$ and a volume $V$ is given by the sum of the following two contributions, i.e., $F=F_{1}+F_{2}$. The first contribution is the sum of the bending energy and the energy coming from the osmotic pressure difference $P=P_{\text {in }}-P_{\text {out }}$ between the inside and outside of the vesicle, which can be expressed as [7, 23, 24]

$$
\begin{aligned}
F_{1}= & \int_{S_{\mathrm{o}}} \frac{1}{2} \kappa(\phi)\left[H-H_{\mathrm{sp}}(\phi)\right]^{2} d A \\
& +\int_{S_{\mathrm{o}}} \kappa_{\mathrm{G}}(\phi) K d A+P \int_{V} d V
\end{aligned}
$$

where $H / 2$ and $K$ are the mean and Gaussian curvatures, respectively. $d A$ is an areal element, which is expressed as $d A=\sqrt{g} d u^{1} d u^{2}$ using the metric $g$ of the membrane surface. In a two-component membrane, the local bending rigidities may depend on the local composition in lipid. Assuming that the bending rigidities are linear functions of the local compositions, the bending rigidities can be 
expressed as

$$
\begin{aligned}
\kappa(\phi) & =\kappa^{(\mathrm{A})} \phi_{\mathrm{A}}+\kappa^{(\mathrm{B})} \phi_{\mathrm{B}} \\
\kappa_{\mathrm{G}}(\phi) & =\kappa_{\mathrm{G}}^{(\mathrm{A})} \phi_{\mathrm{A}}+\kappa_{\mathrm{G}}^{(\mathrm{B})} \phi_{\mathrm{B}}
\end{aligned}
$$

where $\kappa^{(a)}$ and $\kappa_{\mathrm{G}}^{(a)}$ are the bending modulus and the saddle splay modulus, respectively, of a membrane made only of a/an $a$-component lipid ( $a=\mathrm{A}$ or B). The spontaneous curvature may also depend on the local composition, and is assumed to be given by

$$
H_{\mathrm{sp}}(\phi)=H_{\mathrm{sp}}^{(\mathrm{A})} \phi_{\mathrm{A}}+H_{\mathrm{sp}}^{(\mathrm{B})} \phi_{\mathrm{B}}
$$

where $H_{\mathrm{sp}}^{(a)}$ is the spontaneous curvature of a membrane consisting only of a/an $a$-component lipid. The second contribution is a mixing free energy describing the intramembrane phase separations of the lipids. The mixing free energy is expressed by

$$
\begin{aligned}
& F_{2}=\epsilon_{\mathrm{o}} \int_{\mathcal{S}}\left[\frac{\xi^{2}}{2} g^{\alpha \beta} \phi,{ }_{\alpha} \phi, \beta+f(\phi)\right] d A \\
& f(\phi)=\frac{a_{2}}{2} \phi^{2}+\frac{a_{4}}{4} \phi^{4}
\end{aligned}
$$

where $g^{\alpha \beta}$ is the contravariant metric tensor, defined as the inverse of $g_{\alpha \beta}$, and $\xi$ is proportional to the interface thickness of the domain boundary. The symbol $\epsilon_{\mathrm{o}}$ denotes an area energy density and is set to unity. In eq.(5) and hereafter, $X,{ }_{\alpha}$ stands for a derivative of $X(u)$ with respect to $u^{\alpha}(\alpha \in\{1,2\})$. The first and second terms in the integrand of $F_{2}$ are related to the line energy of the domain boundary and to the mixing free energy density in bulk, respectively. In the bulk mixing energy density of eq.(6), $a_{2}=a\left(T-T_{\mathrm{c}}^{(\mathrm{o})}\right)$ and $a_{4}$ is a positive constant, where $T$ and $T_{\mathrm{c}}^{(\mathrm{o})}$ represent temperature and the bare critical temperature, respectively. The actual critical temperature can be shifted from $T_{\mathrm{c}}^{(\mathrm{o})}$ to a higher temperature by a coupling of the local composition and mean curvature[11]. Using the total free energy $F$, the chemical potential $\mu$ for $\phi$ is given by

$$
\begin{aligned}
\mu= & -\xi^{2} \Delta_{\mathrm{LB}} \phi+a_{2} \phi+a_{4} \phi^{3}+\frac{1}{2} \kappa_{\mathrm{G}}^{\prime} K \\
& +\frac{1}{2} \kappa^{\prime}\left(H-H_{\mathrm{sp}}\right)^{2}-\kappa(\phi)\left(H-H_{\mathrm{sp}}\right) H_{\mathrm{sp}}^{\prime}
\end{aligned}
$$

where the symbols with a prime are derivatives with respect to $\phi$ :

$$
\begin{aligned}
& \kappa^{\prime}=\left(\kappa^{(\mathrm{A})}-\kappa^{(\mathrm{B})}\right) / 2, \\
& \kappa_{\mathrm{G}}^{\prime}=\left(\kappa_{\mathrm{G}}^{(\mathrm{A})}-\kappa_{\mathrm{G}}^{(\mathrm{B})}\right) / 2, \\
& H_{\mathrm{sp}}^{\prime}=\left(H_{\mathrm{sp}}^{(\mathrm{A})}-H_{\mathrm{sp}}^{(\mathrm{B})}\right) / 2 .
\end{aligned}
$$

Here we consider the dynamics of a two-component vesicle, i.e., the phase separation dynamics of lipids and shape deformation dynamics. The phase separation dynamics can be described by the time-dependent Ginzburg-Landau equation in twodimensional curved/curving space. The covariant component $j_{\alpha}^{(\mathrm{T})}$ of the total flux of $\phi$ is given by the sum of the following three contributions (i) random flux $j_{\alpha}^{(\mathrm{R})}$ of $\phi$ from thermal fluctuations, (ii) flux coming from the hydrodynamic convection of $\phi$, (iii) thermodynamic flux induced by a chemical potential gradient $\mu$ for $\phi$. This component is expressed as

$$
j_{\alpha}^{(\mathrm{T})}(u, t)=j_{\alpha}^{(\mathrm{R})}(u, t)+\phi v_{\alpha}-L \mu,_{\alpha}
$$

where $L$ is a transport coefficient and is assumed to be constant. The random flux obeys the fluctuationdissipation theorem and therefore satisfies the following relation

$$
\begin{aligned}
& \left\langle j_{\alpha}^{(\mathrm{R})}(u, t) j_{\beta}^{(\mathrm{R})}\left(u^{\prime}, t^{\prime}\right)\right\rangle= \\
& 2 k_{\mathrm{B}} T L \delta_{\alpha \beta} \frac{1}{\sqrt{g}} \delta\left(u-u^{\prime}\right) \delta\left(t-t^{\prime}\right)
\end{aligned}
$$

where $\langle(\cdots)\rangle$ stands for a statistical average of $(\cdots)$ and $\delta\left(u-u^{\prime}\right) \equiv \delta\left(u^{1}-u^{\prime 1}\right) \delta\left(u^{2}-u^{\prime 2}\right)$. The time-evolution equation for $\phi$ can be obtained from the equation of continuity using eq.(11) for the total flux of $\phi$ as

$$
\frac{\partial}{\partial t} \phi(u, t)=-\left.\left(\phi v_{\alpha}\right)\right|^{\alpha}+L \Delta_{\mathrm{LB}} \mu-\left.j_{\alpha}^{(\mathrm{R})}\right|^{\alpha}
$$

where $\left.X_{\alpha}\right|^{\alpha}=\left.X_{\alpha}\right|_{\beta} g^{\alpha \beta},\left.X_{\alpha}\right|_{\beta}$ denotes a covariant derivative of $X_{\alpha}$ with respect to $u_{\beta}$, and is defined by $\left.X_{\alpha}\right|_{\beta}=$ $X_{\alpha, \beta}-\Gamma_{\alpha \beta}{ }^{\gamma} X_{\gamma}$, where $\Gamma_{\alpha \beta}^{\gamma}$ is the Christoffel symbol defined by $\Gamma_{\alpha \beta}^{\gamma}=\boldsymbol{g}_{\alpha, \beta} \cdot \boldsymbol{g}^{\gamma}$. The symbol $\Delta_{\mathrm{LB}}$ stands for the Laplace-Beltrami operator, which is defined as $\left.\Delta_{\mathrm{LB}} X \equiv X\right|_{\alpha \beta} g^{\alpha \beta}=\left.\left.X\right|_{\alpha}\right|_{\beta} g^{\alpha \beta}=X,\left._{\alpha}\right|_{\beta} g^{\alpha \beta}$. The hydrodynamic velocity field $v_{\alpha}(u, t)$ is defined only on the membrane and can be described by the following Stokes equation in two-dimensional curved space as

$$
\rho \frac{\partial}{\partial t} v_{\alpha}(u, t)=\left.\sigma_{\alpha \beta}^{(\mathrm{v})}\right|^{\beta}-p,{ }_{\alpha}+f_{\alpha}^{(\mathrm{s})}-\phi \mu,,_{\alpha}+\left.\sigma_{\alpha \beta}^{(\mathrm{R})}\right|^{\beta}
$$

where $\rho$ is the density of the fluid membrane, $\left.\sigma_{\alpha \beta}^{(\mathrm{v})}\right|^{\beta}$ is the viscous stress of the fluid membrane and $p$ is the lateral pressure defined only on the surface. Because the membrane is considered to be a two-dimensional viscous fluid, the viscous stress can be expressed as

$$
\sigma_{\alpha \beta}^{(\mathrm{v})}=\eta\left(\left.v_{\alpha}\right|_{\beta}+\left.v_{\beta}\right|_{\alpha}\right)
$$

where $\eta$ is the viscosity of the fluid membrane. Although the viscosity of a fluid membrane, may generally depend on the local composition, i.e., $\eta=\eta(\phi)$, hereafter, we assume that the viscosity of the membrane is constant for simplicity. $\sigma_{\alpha \beta}^{(\mathrm{R})}$ in the last term of eq.(14) is a random stress coming from a thermal fluctuation and satisfies the following relationship [33]

$$
\begin{aligned}
& \left\langle\sigma_{\alpha \beta}^{(\mathrm{R})}(u, t) \sigma_{\mu \nu}^{(\mathrm{R})}\left(u^{\prime}, t^{\prime}\right)\right\rangle \\
& \quad=2 k_{\mathrm{B}} T \eta\left(\delta_{\alpha \beta} \delta_{\mu \nu}+\delta_{\alpha \mu} \delta_{\beta \nu}\right) \delta\left(u-u^{\prime}\right) \delta\left(t-t^{\prime}\right)
\end{aligned}
$$


Because the vesicle is immersed in a viscous solvent, the intra-membrane hydrodynamic flow is affected by a three dimensional hydrodynamic flow of solvent. The incorporation of the hydrodynamic effect of the surrounding solvent on the intra-membrane velocity field $v_{\alpha}$ has already been investigated $[30-32]$. The third term $f_{\alpha}^{(\mathrm{s})}$ in l.h.s of eq.(14) represents a force density exerted on the membrane by the surrounding fluid. In other words, the term describes a momentum leak of the fluid membrane to a surrounding viscous solvent [30-32]. In the present system, the surrounding solvent as a whole is assumed to be in a quiescent state, and the force $f_{\alpha}^{(\mathrm{s})}$ can be expressed by $f_{\alpha}^{(\mathrm{s})}=-\lambda v_{\alpha}$ with a phenomenological dumping parameter $\lambda$. Therefore, the rate of decrease of the momentum density of an intra-membrane hydrodynamic flow per unit time is $-\lambda v_{\alpha}$. Furthermore, the two-dimensional fluid can be regarded as a laterally incompressible fluid. The incompressible condition for a two-dimensional fluid is expressed as

$$
\left.v_{\alpha}\right|^{\alpha}=0 \text {. }
$$

Neglecting the inertia term in eq.(14) and using eq.(17), the equation of the velocity field (14) can be reduced to the following equation

$$
\eta \Delta_{\mathrm{LB}} v_{\alpha}(u, t)-p,{ }_{\alpha}-\lambda v_{\alpha}-\phi \mu,,_{\alpha}+\left.\sigma_{\alpha \beta}^{(\mathrm{R})}\right|^{\beta}=0 .
$$

The equation for the lateral pressure $p$ can be obtained by applying the incompressible condition eq.(17) to eq.(18) and is found to be

$$
\Delta_{\mathrm{LB}} p=-\left.(\phi \mu, \alpha)\right|^{\alpha}+\left.\sigma_{\alpha \beta}^{(\mathrm{R})}\right|^{\alpha \beta} .
$$

Assuming that the membrane moves to reduce the total free energy $F$, the shape-deformation dynamics can be described by an energy relaxation type equation. The time-evolution equation is given by

$$
\begin{aligned}
\frac{\partial \boldsymbol{r}(u, t)}{\partial t} & =-L_{r} \frac{\delta}{\delta \boldsymbol{r}(u, t)}\left\{F+\int \gamma \sqrt{g} d^{2} u\right\}+\boldsymbol{f}^{(\mathrm{R})} \\
& =-L_{r}\left[A_{\alpha} \boldsymbol{g}^{\alpha}+A_{\perp} \boldsymbol{n}\right]+\boldsymbol{f}^{(\mathrm{R})}
\end{aligned}
$$

where $L_{r}$ is a dumping coefficient and $\boldsymbol{f}^{(\mathrm{R})}(u, t)$ is a random force coming from thermal noise. The $i$-th component of the random force $f_{i}^{(\mathrm{R})}$ in a Cartesian coordinate obeys the fluctuation-dissipation theorem and satisfies the following relationship as

$$
\begin{aligned}
& \left\langle f_{i}^{(\mathrm{R})}(u, t) f_{j}^{(\mathrm{R})}\left(u^{\prime}, t^{\prime}\right)\right\rangle \\
& \quad=2 k_{\mathrm{B}} T L_{r} \delta_{i j} \delta\left(u-u^{\prime}\right) \delta\left(t-t^{\prime}\right)
\end{aligned}
$$

where $(i, j \in\{x, y, z\})$ A local incompressibility condition of the membrane area holds because we consider dynamics on a longer time scale than the characteristic relaxation time of local area fluctuations from their preferred areas. Therefore, we introduce a local Lagrange multiplier $\gamma(u)$ that serves to guarantee the incompressibility of the local membrane area. The tangential force
$A_{\alpha}$ and the normal force to the surface $A_{\perp}$ are given as $[34]$

$$
\begin{aligned}
A_{\alpha}= & \left.\xi^{2}(\phi, \alpha \phi, \beta)\right|^{\beta}+B H,_{\alpha}-\bar{\gamma}, \alpha+\kappa_{\mathrm{G}}(\phi) K,_{\alpha} \\
A_{\perp}= & -\xi^{2} b^{\alpha \beta} \phi,_{\alpha} \phi, \beta+P \\
& -B\left(H^{2}-2 K\right)-\Delta_{\mathrm{LB}} B+\bar{\gamma} H \\
& -2 H\left[\kappa_{\mathrm{G}}(\phi) K+\Delta_{\mathrm{LB}} \kappa_{\mathrm{G}}(\phi)\right]+\left.\kappa_{\mathrm{G}}(\phi)\right|_{\alpha \beta} b^{\alpha \beta}
\end{aligned}
$$

where $B=\kappa(\phi)\left[H-H_{\mathrm{sp}}(\phi)\right]$ and $\bar{\gamma}=\gamma+\left(1 / 2 \kappa H^{2}+\right.$ $\left.\kappa_{\mathrm{G}} K\right)+\left[\xi^{2} / 2(\nabla \phi)^{2}+f(\phi)\right]$. The equation for $\bar{\gamma}$ can be obtained from the local incompressibility condition, i.e., $\partial \sqrt{g} / \partial t=0$, and is

$$
\left(\Delta_{\mathrm{LB}}-H^{2}\right) \bar{\gamma}-C=0
$$

where $C$ is defined as

$$
\begin{aligned}
C=-H[ & \xi^{2} b^{\alpha \beta} \phi,^{\alpha} \phi,^{\beta} \\
& +B\left(H^{2}-2 K\right)+\Delta_{\mathrm{LB}} B \\
& \left.+2 H\left(\kappa_{\mathrm{G}} K+\Delta_{\mathrm{LB}} \kappa_{\mathrm{G}}\right)-\left.\kappa_{\mathrm{G}}\right|_{\alpha \beta} b^{\alpha \beta}\right]-P \\
+ & \left.\xi^{2}\left(\phi,{ }^{\alpha} \phi,{ }^{\beta}\right)\right|_{\alpha \beta}+\left.\left(A H,{ }^{\alpha}\right)\right|_{\alpha}+\left.\left[\kappa_{\mathrm{G}}(\phi) K,{ }^{\alpha}\right]\right|_{\alpha} .
\end{aligned}
$$

The equations that need to be solved to investigate the dynamics of intra-membrane phase separation and vesicle shape deformation are eqs.(13), (21) and (25).

\section{NUMERICAL SIMULATION}

\section{A. Thermal noise effect on phase separation for a spherical vesicle}

Before investigating the full dynamics of a twocomponent vesicle in which the shape deformation and the intra-membrane phase separation interact, we examine the effects of (i) thermally induced random flux, (ii) intra-membrane hydrodynamic flow and (iii) the composition-dependent bending rigidity $\kappa(\phi)$ on the intra-membrane phase separation dynamics by numerically solving eq.(13) on a vesicle with a fixed shape. Assuming that a vesicle has very little excess area and that the exchange rate of water between the inside and the outside of the vesicle through the membrane is much slower than the inverse of the characteristic diffusion time of the lipid, we can use a rigid sphere as the fixed shape of vesicle to investigate the effect of (i) the thermal noise and (ii) the hydrodynamic flow. 


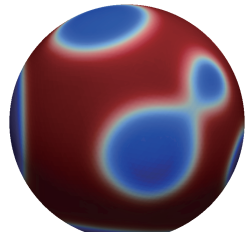

(a-1) $\tilde{\mathrm{t}}=100$

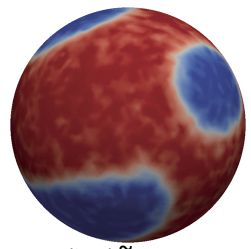

(b-1) $\tilde{t}=100$

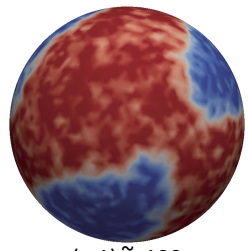

(c-1) $\tilde{t}=100$

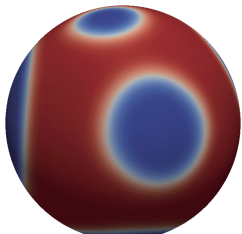

(a-2) $\tilde{t}=300$

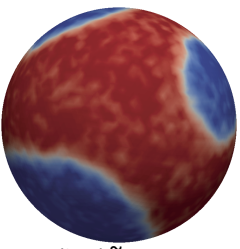

(b-2) $\tilde{\mathrm{t}}=300$

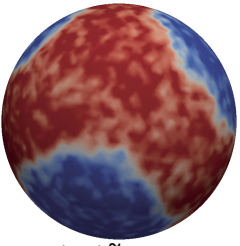

(c-2) $\tilde{\mathrm{t}}=300$

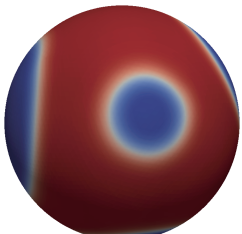

(a-3) $\tilde{\mathrm{t}}=500$

(b-3) $\tilde{\mathrm{t}}=500$

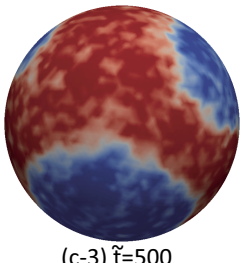

(c-3) $\tilde{t}=500$

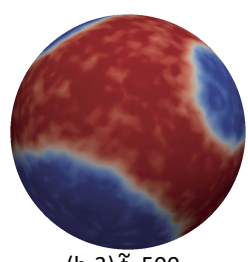

FIG. 2: Typical patterns of phase separation in $\phi_{\mathrm{o}}=0.3$ for a perfect spherical vesicle for (a) $\zeta=0$ (without thermal random flux), (b) $\zeta=0.4$, and (c) $\zeta=0.8$ at $\tilde{t}=100, \tilde{t}=300$, and $\tilde{t}=500$, where $\phi_{\mathrm{o}}$ are defined as the spatial average of $\phi$.

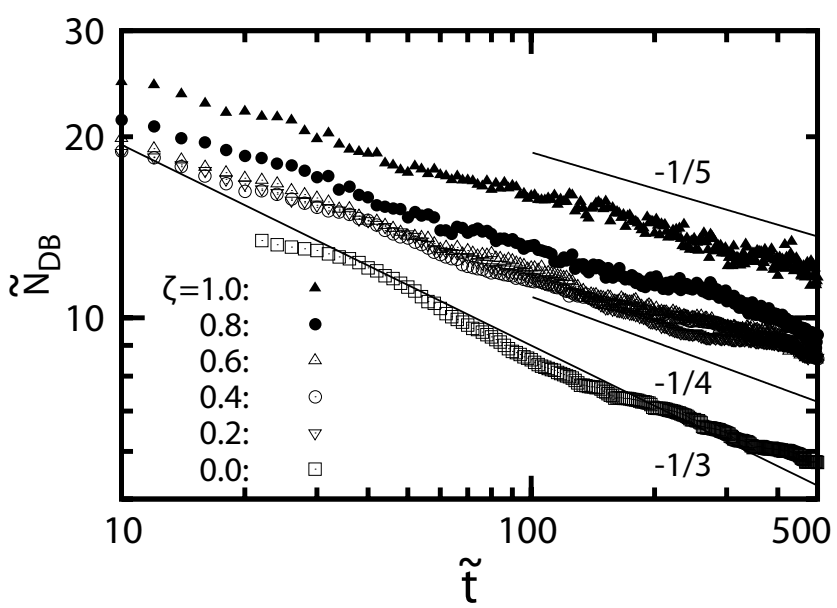

FIG. 3: Log-log plot of $\tilde{N}_{\mathrm{DB}}$ versus the scaled time $\tilde{t}$. The plot for $\zeta=0.0(\square)$ is shifted downward to avoid overlapping the other graphs of $\zeta \neq 0$. The solid lines are guides $\tilde{N}_{\mathrm{DB}} \simeq t^{-1 / 3}$, $t^{-1 / 4}$ and $t^{-1 / 5}$ from the bottom to top, respectively. The data for each $\zeta$ are averaged over five independent runs.

We also use an end-capped cylinder to clarify (iii) the effect of the composition-dependent bending rigidity $\kappa(\phi)$ on the phase separation dynamics. We take the unit of space to be $\tilde{\xi}\left(\equiv \xi /\left|a_{2}\right|^{1 / 2}\right)$ and the unit of time $\tau$ to be $\tau=\tilde{\xi}^{2} / L\left|a_{2}\right|$. We scale $\phi$ by $\phi_{\text {eq }}=\left(\left|a_{2}\right| / a_{4}\right)^{1 / 2}$. Us- ing the space and time units, the strength of the random flux in eq.(11) can be specified by $\zeta$, defined as $\zeta=\left[2 k_{\mathrm{B}} T a_{4} / \tilde{\xi}^{2}\left|a_{2}\right|^{2}\right]^{1 / 2}$. In Fig. 2, we observe typical patterns of intra-membrane phase separation in $\phi_{\mathrm{o}}=0.3$ with different strengths of thermal noise (a) $\zeta=0$ (no thermal noise) (b) $\zeta=0.4$ and (c) $\zeta=0.8$ at the scaled time $\tilde{t}=100,300$ and 500 , where $\phi_{\mathrm{o}}$ is defined as the spatial average of $\phi$. We can see from Fig. 2 that the distortion of the domain boundary increases with an increase in the strength of the random flux. This trend implies that the effective line tension is reduced by the thermal noise. The distorted domain boundary and reduction of effective line tension may influence the coarsening dynamics of the phase-separated pattern. In Fig. 3 , we show a log-log plot of $\tilde{N}_{\mathrm{DB}}(t)$, which is defined as $\tilde{N}_{\mathrm{DB}}(t)=100 N_{\mathrm{DB}}(t) / N_{\text {Total }}$, where $N_{\mathrm{DB}}$ is the number of lattice points located at the domain boundary $(\phi \simeq 0)$ and $N_{\text {Total }}$ is the total number of lattice points. The data for $\zeta=0$ begin at $\tilde{t}=20$ in Fig. 3 because the domain boundary has not yet been established for $\tilde{t}<20$, as seen in Fig. 4. Figure 4 shows the standard deviation of

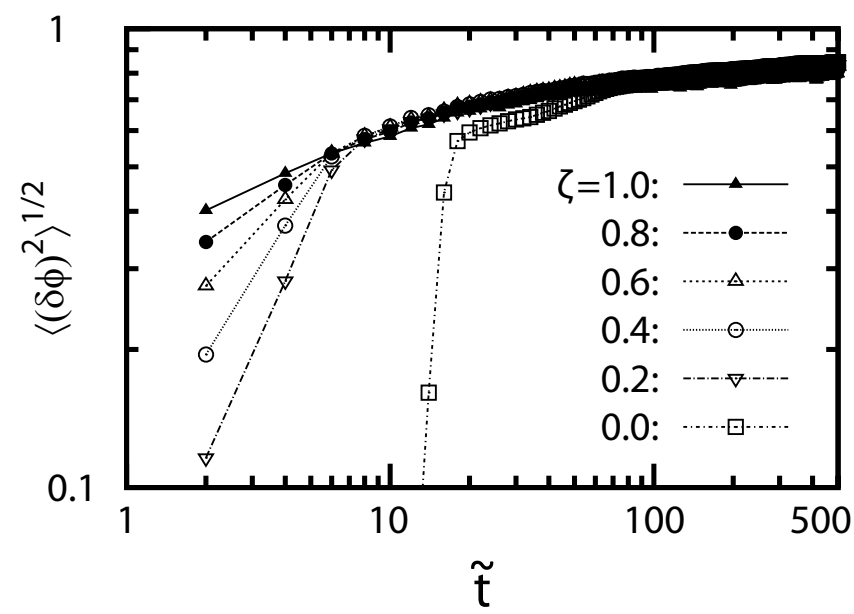

FIG. 4: The standard deviation of $\phi$ from the average value $\phi_{\mathrm{o}}=0.3$ as a function of $\tilde{t}$. The data for each $\zeta$ are averaged over five independent runs.

$\phi$ from the average value $\phi_{\mathrm{o}}=0.3$ as a function of $\tilde{t}$,

$$
\left\langle(\delta \phi)^{2}\right\rangle \equiv \frac{1}{S_{\mathrm{o}}} \int\left[\phi(u, t)-\phi_{\mathrm{o}}\right]^{2} d A,
$$

where $S_{\text {o }}$ is the total surface area of the vesicle. In the early stage of phase separation, the thermally induced random current enhances the growth of $|\delta \phi|$ with an increase of $\zeta$, as seen in Fig. 4. Regarding to the coarsening dynamics in the late stage, as clearly seen in Fig. 3, we have confirmed that $N_{\mathrm{DB}}$ follows the " $(-1 / 3)$-power law" (i.e., $z=1 / 3, N_{\mathrm{DB}} \sim t^{-z}$ ) for $\zeta=0.0$ [12], although the coarsening slows after $\tilde{t} \simeq 300$ because the domain size almost reaches the system size. On the other hand, when $\zeta \geq 0.1$ the coarsening is slower than for $\zeta=0$ because of a thermal-noise effect. The dynamical exponent 
$z$ when $\zeta \geq 0.1$ is $1 / 5<z<1 / 4$. The decrease of $z$ in $\zeta \geq 0.1$ may result from a significant decrease in the effective line tension due to the thermal noise. So far, the investigated coarsening dynamics in off-critical quenches have had phase-separated patterns that are a dispersion of circular domains, and the total length of their domain boundaries decreases as $t^{-1 / 3}[12-17]$. It has been generally accepted that the coarsening dynamics in off-critical quenches can be explained by the following two different mechanisms: as (i) collision and coalescence of domains migrating with Brownian motion and (ii) LifshitzSlyozov ("evaporation-condensation") processes. As already known for the phase separation in bulk systems, Lifshitz-Slyozov mechanisms give $z=1 / 3$, and the Brownian coagulation process gives $z=1 / d[35]$, where $d$ is the system dimensionality. If these assumption are valid in the phase separation on the sphere surface, the exponent should be $z=1 / 2$ in the dominant Brownian coagulation process case and $z=1 / 3$ with no thermal noise. Because there is no Brownian migration of a circular domain for $\zeta=0$ (see Fig. 2(a)), the main mechanism of growth, especially in the late stage, is (ii) the LifshitzSlyozov mechanism. Precisely speaking, however, we can see some domain-coalescence events in the early and intermediate stages. These domain coalescence are induced not by the Brownian migrations of domains but by migrations induced by a composition gradient because the distances between adjacent domains in the early and intermediate stages are small. Such an event can be seen in the phase-separated pattern at $\tilde{t}=100$ in Fig. 2(a) $(\zeta=0.0)$. We also see an evaporation of a domain located near the north pole of the sphere and a ripening of the domain located at the left side of the sphere in Fig. 2(a). Therefore, in the early and intermediate stages (i') domain coalescence through migrations induced by a composition gradient (not by Brownian motion) and (ii) a Lifshitz-Slyozov mechanism comprise the coarsening mechanism. In late stage, on the other hand, the (ii) Lifshitz-Slyozov mechanism is the main mechanism, although still there is a small possibility of observing the composition-gradient-induced coalescence even in the late stage. In cases with $\zeta \neq 0$, we can observe Brownian domain migrations, domain coalescence and evaporationcondensation processes. As seen from the snapshots for $\zeta=0.4$ and $\zeta=0.8$ in Fig. 2(b) and (c), it takes longer for collision and coalescence of circular domains by Brownian migration to occur.

\section{B. Hydrodynamic effects on phase separation on a spherical vesicle}

Next we demonstrate the effects of intra-membrane hydrodynamic flow induced by a chemical potential gradient (i.e., the term in eq.(18), $-\phi \mu,{ }_{\alpha}$, involving an interfacial stress at a domain boundary) on the phase separation dynamics. The flow field is obtained by solving eqs.(13), (18) and (19). To make eq.(18) and eq.(19) dimensionless using units of time and space, $v_{\alpha}, p$ and $\mu$ are scaled by $v_{\mathrm{o}}=\xi / \tau, \sigma_{\mathrm{o}}=\eta / \tau$ and $\mu_{\mathrm{o}}=\left|a_{2}\right| \phi_{\text {eq }}$, respectively. The dimensionless parameters appearing in the dimensionless expressions of eq.(18) and eq.(19) are $\tilde{\lambda}=\lambda /\left(\eta / \xi^{2}\right)$ and $\tilde{g}=\tau\left|a_{2}\right|^{2} / \eta a_{4}$ preceding $\tilde{\phi} \tilde{\mu}, \tilde{\alpha}$. To clarify the hydrodynamic effects on the phase separation dynamics, we omit the thermal noise terms, (i.e., the random current in eq.(13), and random stress in eq.(18)). In Fig. 5, we show a phase-separated pattern and the hydrodynamic flow field in the system of $\phi_{\mathrm{o}}=-0.2, \tilde{\lambda}=0.1$ and $\tilde{g}=1$ at $\tilde{t}=250$. The phase separation pattern shown in Fig. 5 occurs just after the coalescence of two domains, and a flow field is induced to reduce the length of the domain boundary. Similar to a binary fluid blend in a bulk system, the effect of intra-membrane hydrodynamic flow may influence the coarsening dynamics of the domains. Figure 6 shows a log-log plot of $N_{\mathrm{DB}}$ versus time in the system of $\phi_{\mathrm{o}}=0$ (critical quenching case). If the viscosity of the surrounding solvent is chosen to be high, the value of the dumping parameter $\lambda$ in eq.(14) will be larger, which causes greater suppression of the intramembrane hydrodynamic flow. The effect of the dumping constant $\lambda$ on the coarsening dynamics can be seen in Fig. 6. The time dependences of the total perimeter length $N_{\mathrm{DB}}$ for $\tilde{\lambda}=10$ show a similar behavior to those of the system with no hydrodynamic flow. On the other hand, the rate of decrease of $N_{\mathrm{DB}}$ in the system

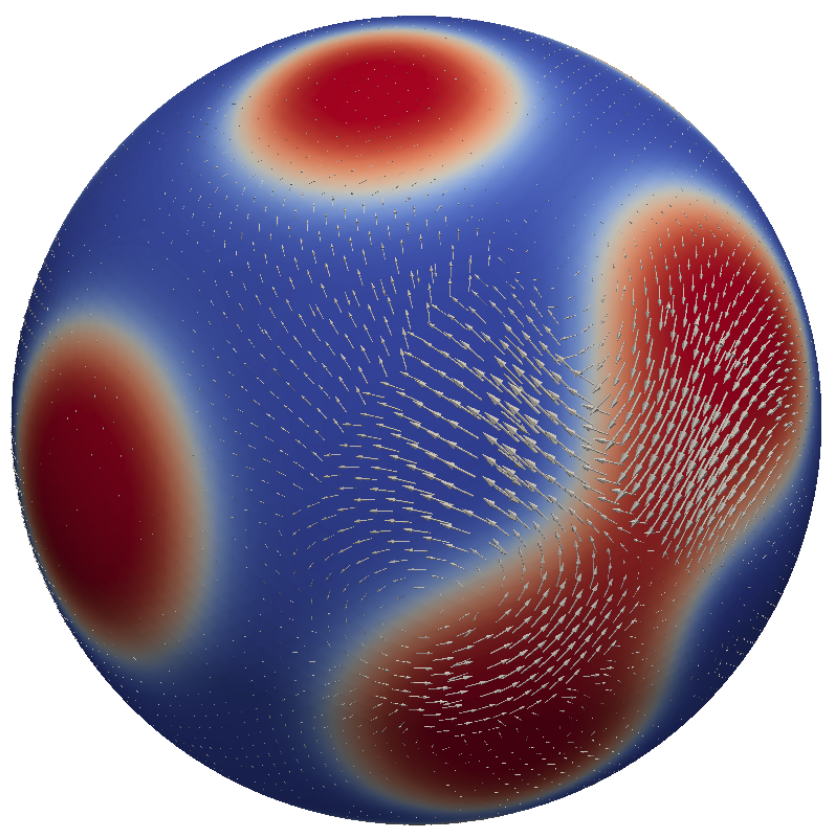

FIG. 5: Phase-separated patterns in the system of $\phi_{\mathrm{o}}=$ -0.2 and $\tilde{\lambda}=0.1$ at $\tilde{t}=250$. The arrows shown on the phase-separated pattern represent an intra-membrane hydrodynamic flow field induced by the line tension at the domain boundary. Note that the flow field is magnified by one hundred times to make the flow field clearly visible. 


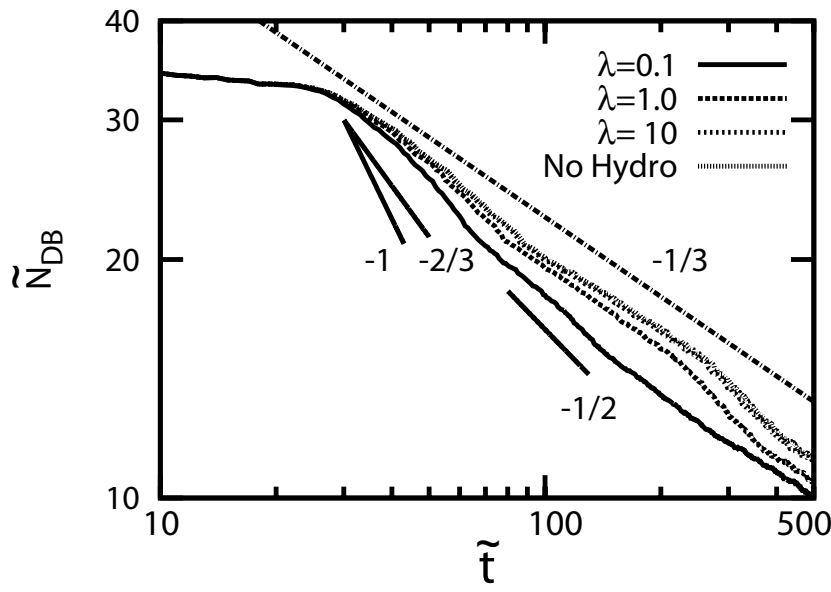

FIG. 6: Log-log plot of $\tilde{N}_{\mathrm{DB}}$ versus $\tilde{t}$ in the system of $\phi_{\mathrm{o}}=0$ for (a) no hydrodynamic flow, (b) $\tilde{\lambda}=10$, (c) $\tilde{\lambda}=1$ and (d) $\tilde{\lambda}=0.1$ and $\tilde{g}=1$. The guide lines $\tilde{N}_{\mathrm{DB}} \simeq t^{-1 / 3}, t^{-1 / 2}, t^{-2 / 3}$ and $t^{-1}$ are also shown. The data for each $\lambda$ are averaged over five independent runs.

of $\tilde{\lambda}=0.1$ becomes faster than that of $\tilde{\lambda} \geq 1$. Figure 7 shows the magnitude of hydrodynamic flow $V_{\text {mag }}$ induced by the line tension of the domain boundary. $V_{\text {mag }}$ is defined by

$$
V_{\mathrm{mag}}=\left[\frac{1}{S_{\mathrm{o}}} \int_{S_{\mathrm{o}}} g^{\alpha \beta} v_{\alpha} v_{\beta} d A\right]^{1 / 2} .
$$

It is clearly seen from Fig. 7 that the hydrodynamic flows are more strongly suppressed with increasing $\tilde{\lambda}$. As seen from Fig. 6, the time region $\tilde{t}<30$ is an early stage, and the coarsening of domains begins. As clearly shown in Fig. 6 , the dynamical exponent $z$ in $\tilde{\lambda}=0.1$

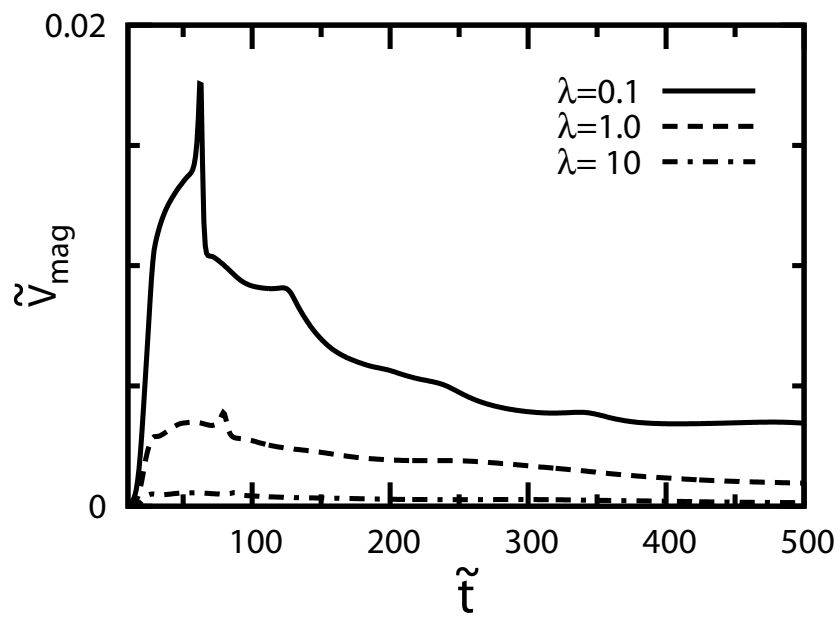

FIG. 7: Magnitude of the velocity field induced by the line tension of domain boundaries appearing in the system of $\phi_{\mathrm{o}}=$ 0 . The system corresponds to that shown in Fig. 6 . is larger than $1 / 3$. In particular, $z$ is close to $2 / 3$ in the $30<\tilde{t}<70$ time region, where the magnitude of hydrodynamic flow defined in eq.(28) becomes large as seen in Fig. 7. In the time region $\tilde{t}>70$, the magnitude of the hydrodynamic flow $V_{\mathrm{mag}}$ is smaller than that in the time region $30<\tilde{t}<70$. The exponent $z$ is close to $1 / 2$ [35] in the time region $70<\tilde{t}<200$, and $z$ approaches $1 / 3$ in $\tilde{t}>300$. The fact that the exponent $z$ is not unity in the time region where the hydrodynamic effect becomes dominant in coarsening dynamics but is close to a value in the range $1 / 2<z<2 / 3$ may be attributed to the dimensionality of the system. It has been reported in two-dimensional bulk systems that the exponent $z$ is $2 / 3$ even in the region where the hydrodynamic effect is dominant [36]. The exponent $z=1 / 3$ in the very late stage (i.e., $\tilde{t}>300$ ) for $\tilde{\lambda}=0.1$ is probably due to a finite system size effect.

C. Effect of composition-dependent bending rigidity on phase separation on a tubular vesicle

To investigate the effects of composition-dependent bending rigidities on phase separation, we perform simulations of phase separation on a shape-fixed tubular vesicle with $\kappa^{(\mathrm{A})} / \kappa^{(\mathrm{B})}=1.25$. The tubular vesicle used in the present work consists of a cylinder with a radius $R_{\mathrm{o}}(\simeq 11.2 \tilde{\xi})$ and a length $L_{\mathrm{o}}(\simeq 39.0 \tilde{\xi})$ and two hemispheres with the same radius $R_{0}$, as shown in Fig. 8(a). Tubular vesicles with a shape similar to the capped cylinder can be realized by imposing a large osmotic pressure difference $[23,24,34]$. To clarify the effects of composition-dependent bending rigidity $\kappa(\phi)$ on phase separation, we fix the shape of the cylindrical vesicle. Although the Gaussian modulus $\kappa_{\mathrm{G}}$ and the spontaneous curvature $H_{\mathrm{sp}}$ may also depend on the local composition, we have neglected their composition dependence to focus only on the effect of $\kappa(\phi)$. We show the time evolutions of phase separation on a tubular vesicle with a constant bending rigidity $\kappa_{\mathrm{o}}$ in Fig. 8 and with a compositiondependent bending rigidity $\kappa(\phi)$ in Fig. 9. We assume that the type-A and type-B lipids are homogeneously distributed in the initial state with a small thermal composition fluctuation in the tubular vesicle. After quenching the system to the region inside the binodal line of phase separation, the usual isotropic spinodal decomposition takes place when the bending rigidity is constant, as seen in Fig. 8. When the bending rigidity depends on the local composition, an axisymmetric band-like phase separation with a periodicity along the longer axis of the tubular vesicle occurs in the early stage $((\mathrm{a}) \tilde{t}=10)$. The phase-separated band structure coarsens with time, as seen in Fig. 9. The formation of the band structure of phase-separated domains comes from the coupling of the composition-dependent bending rigidity and the local mean curvature. The type-A lipid whose membranes have a higher rigidity prefer to diffuse toward the cylinder part with a lower mean curvature and away from 


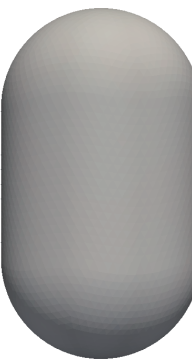

(a) $\tilde{t}=10$

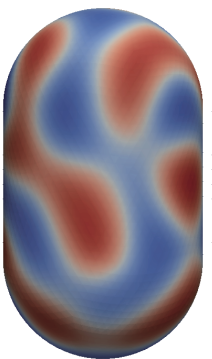

(b) $\tilde{t}=50$

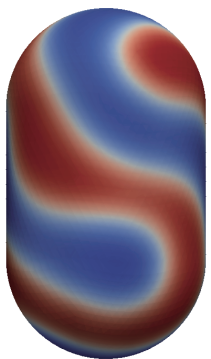

(c) $\tilde{\mathrm{t}}=100$

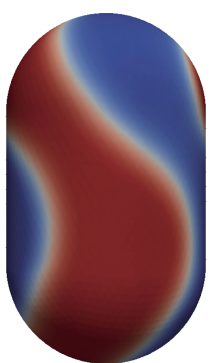

(d) $\tilde{\mathrm{t}}=400$
FIG. 8: Time-sequential snapshots of phase separation on a vesicle with a constant bending rigidity $\kappa_{\mathrm{o}}$ at $\tilde{t}=$ $10,50,100,400$.

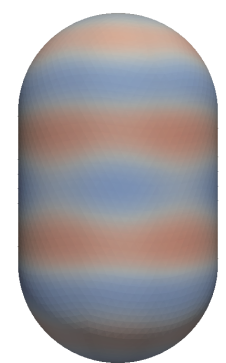

(a) $\tilde{\mathrm{t}}=10$

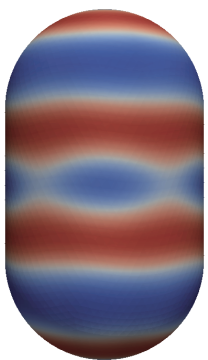

(b) $\tilde{\mathrm{t}}=50$

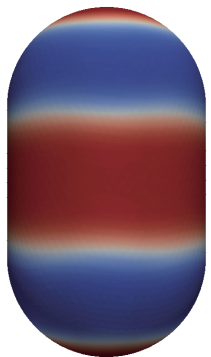

(c) $\tilde{t}=100$

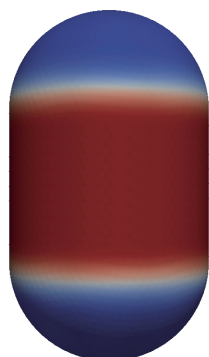

(d) $\tilde{t}=400$
FIG. 9: Time sequential snapshots of phase separation on a vesicle with a composition-dependent bending rigidity $\kappa(\phi)$ at $\tilde{t}=10,50,100,400$.

the hemisphere with a higher mean curvature. Therefore, the type-A lipids near the region that connects the cylinder and hemisphere cap diffuse toward the cylinder part; simultaneously, the type-B lipids with a lower rigidity counter-diffuse toward the caps. However, as seen in Fig. 9(a) and (b), the type-A lipids close to both ends of the capped cylinder are left behind in the beginning of phase separation, although the type-A lipids prefer the flat region. The domains rich in the type-A lipids left behind at the ends of both caps then disappear through the evaporation-condensation process. These phase separation processes explained above occur axisymmetrically, except for coalescence of bands in the coarsening process seen in Fig. 9(b), which is the origin of the formation of the band structure for $\kappa=\kappa(\phi)$.

\section{Effect of composition-dependent bending rigidity $\kappa(\phi)$ on shape deformation and phase separation dynamics}

Using a deformable lattice, we examine the effects of composition-dependent bending rigidity on the dynamics of the shape deformation and intra-membrane phase separation, and compare the numerical results with experimental results obtained by Yanagisawa et al [23, 24].
In these experiments, the shapes of vesicle are first controlled by an applied osmotic pressure difference at a temperature higher than $T_{\mathrm{c}}$, where the two-component membrane exhibits a single phase, and the temperature of the system is then quenched to induce an intramembrane phase separation. They found a wide variety of shape deformations coupled with phase separations. In addition, various shapes induced by osmotic pressure difference[37], e.g., prolate-, oblate-, tripod- and starfish-like shapes, converge to oblate shapes after intramembrane phase separation, which is referred to as shape convergence. In our numerical simulations, we follow the same procedure as that performed in the experiments. By solving eq.(21) in homogeneous vesicles over a long time so that the systems reach a stable state under an applied osmotic pressure difference $\tilde{P}=P r_{\mathrm{o}}^{3} / \kappa_{\mathrm{o}}=8.5$, we obtained (a) biconcave and (b) prolate dumbbell-shaped vesicles (see Fig. 10(a) and (b)) as a metastable state corresponding to an energy minimal state and an equilibrium state with minimal of free energy $F_{1}$, respectively $[11,34]$. Next, we quench systems with these two shapes and with various $\phi_{\mathrm{o}}$ 's as initial states to a temperature lower than $T_{\mathrm{c}}$ to invoke a phase separation, and we investigate the dynamics of shape deformation and phase separation.

First, in Fig. 11 we show stable shapes and phaseseparated patterns obtained by long-time numerical simulations for $\phi_{\mathrm{o}}=-0.2,0$ and 0.2 where the biconcave shape shown in Fig. 10(a) is used as the initial shape. As seen in Fig. 11, when the initial shape is biconcave, the obtained stable shapes in $\phi_{\mathrm{o}}=0$ and $\phi_{\mathrm{o}}=-0.2$ are pancake-like. The stable shape for $\phi_{\mathrm{o}}=0.2$, on the other hand, is prolate. The characteristic feature of these stable states in Fig. 11 is that each of these stable shapes is composed of two less-curved end-caps rich in the type-A lipid and a cylinder rich in the typeB lipids. Dynamical processes to reach the stable states are qualitatively same and are explained below. After the quenching, first the type-A lipids diffuse to the top

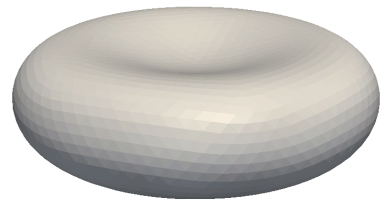

(a)

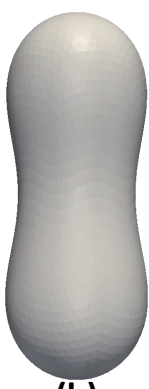

(b)
FIG. 10: Initial shapes used in the simulation, (a) biconcave and (b) prolate dumbbell shapes. These initial shapes (a) and (b) are stationary and at equilibrium, satisfying $A_{\perp}=0$ in eq. (24) with $\tilde{P}=8.5$. 


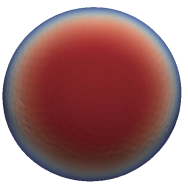

(a) $\phi_{0}=-0.2$ (Top)

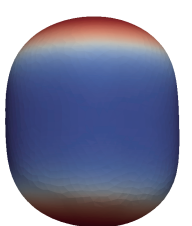

(a) $\phi_{\mathrm{o}}=-0.2$ (Side)

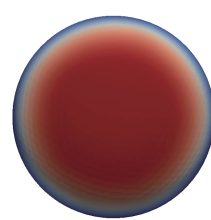

(b) $\phi_{\mathrm{o}}=0$ (Top)

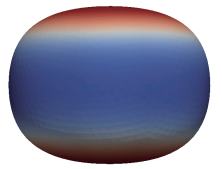

(b) $\phi_{\mathrm{o}}=0$ (Side)

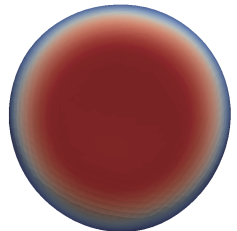

(c) $\phi_{0}=+0.2$ (Top)

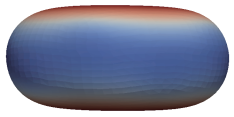

(c) $\phi_{\mathrm{o}}=+0.2$ (Side)
FIG. 11: Stable shapes (top views in the upper line and side views in the bottom line) and the phase-separated pattern obtained in the system (a) $\phi_{\mathrm{o}}=-0.2$, (b) $\phi_{\mathrm{o}}=0$ and (c) $\phi_{\mathrm{o}}=0.2$ after phase separation using the biconcave shape shown in Fig. 10(a) as the initial state.
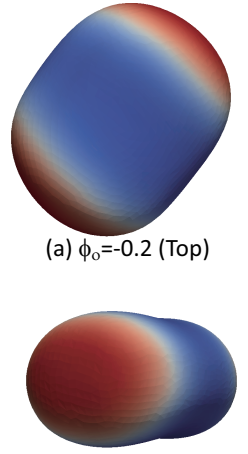

(a) $\phi_{\mathrm{o}}=-0.2$ (Side)

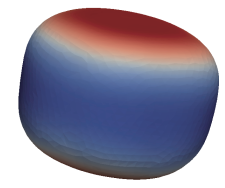

(b) $\phi_{\mathrm{o}}=0$ (Top)

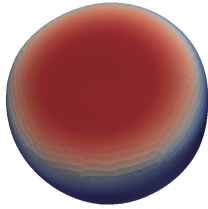

(b) $\phi_{\mathrm{o}}=0$ (Side)

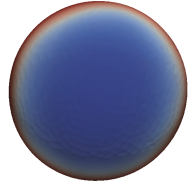

(c) $\phi_{o}=+0.2$ (Top)

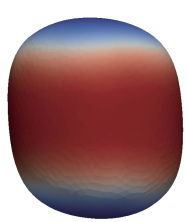

(c) $\phi_{\mathrm{o}}=+0.2$ (Side)
FIG. 12: Stable shapes (top views in the upper line and side views in the bottom line) and phase separated pattern obtained in the system (a) $\phi_{\mathrm{o}}=-0.2$, (b) $\phi_{\mathrm{o}}=0$ and (c) $\phi_{\mathrm{o}}=0.2$ after phase separation using the prolate dumbbell shape shown in Fig. 10(b) as the initial state.

and bottom concave regions where the mean curvature is smaller than that in the other region as demonstrated in the phase separation on the fixed tubular vesicle in the section III C. The concave shapes in the two regions then change to almost flat shapes. The shape deformation and phase separation described above occur axisymmetrically, which results in the states in Fig. 11. The radius of the nearly flat end-cap of these stable shapes increases with $\phi_{\mathrm{o}}$ as $r_{\mathrm{o}}\left(1+\phi_{\mathrm{o}}\right)^{1 / 2}$ and equivalently the length of cylindrical domain decreases with the increase of $\phi_{\mathrm{o}}$ as $r_{\mathrm{o}}\left(1-\phi_{\mathrm{o}}\right) /\left(1+\phi_{\mathrm{o}}\right)^{1 / 2}$, where $r_{\mathrm{o}}=\left(S_{\mathrm{o}} / 4 \pi\right)^{1 / 2}$.

In Fig. 12 we also show stable shapes and phaseseparated patterns obtained by the same procedure as
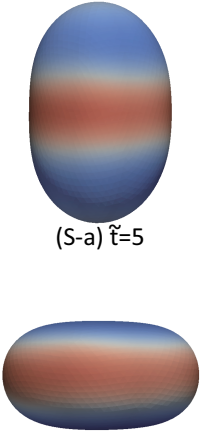

(S-d) $\tilde{t}=35$

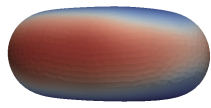

(S-g) $\tilde{\mathrm{t}}=500$

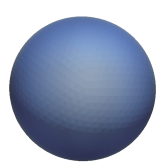

(T-a) $\tilde{t}=5$

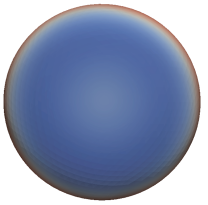

(T-d) $\tilde{t}=35$

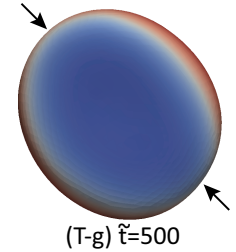

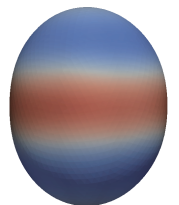

(S-b) $\tilde{t}=10$

(S-e) $\tilde{\mathrm{t}}=65$

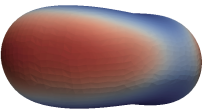

(S-h) $\tilde{t}=600$

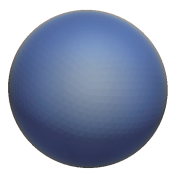

(T-b) $\tilde{t}=10$

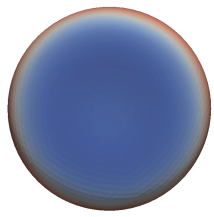

(T-e) $\tilde{\mathrm{t}}=65$

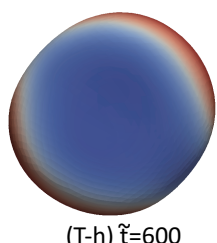

(T-h) $\tilde{t}=600$

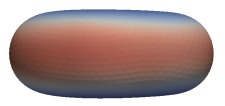

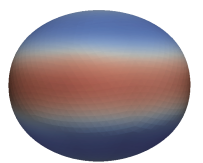

(S-c) $\tilde{t}=20$
FIG. 13: Time sequential snapshots of a system of $\tilde{\kappa}^{\prime}=0.1$ and $\phi_{\mathrm{o}}=-0.2$ at $\tilde{t}=$ (a) 5, (b) 10, (c) 20, (d) 35, (e) 65, (f) 300, (g) 500, (h) 600 and (i) 800 after quenching the temperature below $T_{\mathrm{c}}$. The initial shape $(\tilde{t}=0)$ is the prolate dumbbell shape shown in Fig. 10(b). The top and side views for each time are specified by "T-" and "S-" in the figures.

in Fig. 11, but the initial shape used here is the prolate dumbbell shape shown in Fig. 10(b). As seen from comparison between the states with the same $\phi_{\mathrm{o}}$ in Fig. 11 and Fig. 12, the obtained stable shapes in $\phi_{\mathrm{o}}=-0.2$ and $\phi_{\mathrm{o}}=0$ are basically the same as those in Fig. 11. This finding indicates that the obtained stable states in $\phi_{\mathrm{o}}=-0.2$ and $\phi_{\mathrm{o}}=0$ are the equilibrium states, although these two corresponding shapes (Fig. 11(a) and Fig. 12(a), Fig. 11(b) and Fig. 12(b)) are not exactly 
the same. In the case of $\phi_{\mathrm{o}}=0.2$ shown in Fig. 11(c), however, the stable shape and the phase-separated pattern are different from those in Fig. 12(c) obtained in the case that the initial shape is the biconcave shape. To understand why these two stable states in $\phi_{\mathrm{o}}=0.2$, Fig. 11(c) and Fig. 12(c), are different, in the following we consider how the systems in Fig. 12 reach these stable shapes. Prior to explaining the time evolution of the system shown in Fig. 12(c) $\phi_{\mathrm{o}}=0.2$, we display in Fig. 13 snapshots of the time evolution of a system started from an initial state with $\phi_{\mathrm{o}}=-0.2$ and with the prolate dumbbell shape shown in Fig. 10(b). As seen from Fig. 13(a), the type-A lipids with higher bending rigidity prefer to diffuse to the cylinder region with a mean curvature smaller than that in the cap regions and then form a domain at the cylinder part. After the type-A lipids form the cylindrical domain, the cylinder radius becomes larger and the length of the cylinder simultaneously becomes smaller to reduce the curvature of the cylinder, as seen in (a)-(d) of Fig. 13. The oblate shape seen in (Se) and (T-e) seems to be stable for a certain time up to around $\tilde{t} \simeq 300$, but the circular cross-section of cylinder begins to distort to an ellipsoidal shape from $\tilde{t} \simeq 300$, as can be seen in Fig. 13(T-e). As shown by arrows in Fig. 13(T-f), in the distorted cylinder domain, two regions with higher curvatures and lower concentrations in type-A lipid are formed, and therefore the rigidity of the membrane at the two regions becomes smaller. The two weaker regions against a bend are formed almost simultaneously at the positions in opposition to each other. Then, by breaking the cylinder domain into two domains as seen in (e)-(h), each of the two domains rich in the type-A lipid forms a domain with a less curvature, which results in the state shown in (i). It should be noted that the place where the cylindrical domain breaks into two domains comes from an initially given concentration distribution with a small fluctuation; however, the relative positions of the two places of breaking, will be always in opposition. The state shown in (i) was quite stable for our possible simulation time, and the state is hence considered to be an equilibrium state. In the system with $\phi_{\mathrm{o}}=0$ and the prolate dumbbell shape as the initial shape, we can also see qualitatively the same time evolution as in Fig. 13 for $\phi_{\mathrm{o}}=-0.2$.

In the system of $\phi_{\mathrm{o}}=0.2$, however, the time evolution and the resultant stable state are different from that described above. In this case, the type-A lipids also prefer to diffuse to the cylinder region with a mean curvature smaller than that in the cap regions and form a domain at the cylinder portion described in the case of $\phi_{\mathrm{o}}=-0.2$. However, after forming the cylindrical domain rich in type-A lipids, the cylindrical domain becomes stable. Although the state shown in Fig. 12(c) is considered a metastable state rather than an equilibrium state, the state is quite stable because the cylindrical domain is too large to be broken into two domains to reduce the total bending energy. Actually, the size of the cylindrical domain in $\phi_{\mathrm{o}}=0.2$ is larger than those in

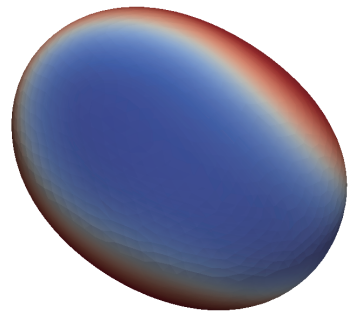

Top View

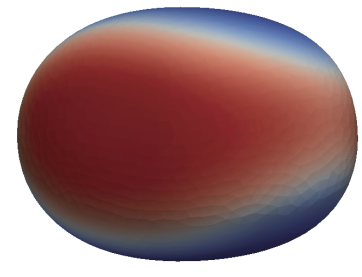

Side View
FIG. 14: Another type of stable state ((a)top views and (b) side views) seen in the system with $\phi_{\mathrm{o}}=0$ and with the prolate dumbbell shape shown in Fig. $10(\mathrm{~b})$ as the initial state. The phase-separated pattern in the stable state is similar to the two leather pieces covering a baseball.

$\phi_{\mathrm{o}}=-0.2$ and $\phi_{\mathrm{o}}=0$. Therefore, within our possible simulation time the system can not reach the state shown in Fig. 11(c), which is considered to be an equilibrium state.

As explained above, after quenching of the systems to induce phase separations, almost all of the states started from the biconcave and prolate dumbbell shapes fall into states composed of two almost flat domains rich in the type-A lipids with higher bending rigidity and a cylindrical domain rich in the type-B lipids, except for the states shown in Fig. 12(c). These results are in good agreement with experimentally observed behaviors known as shape convergence.

In the system of $\phi_{\mathrm{o}}=0$, we also observed another stable state whose shape is close to an ellipsoid and whose phase-separated pattern is similar to the two leather pieces of a baseball, as shown in Fig. 14. The state is also stable because the thermal random current of $\phi$ is neglected in simulations performed in Sec. IIID. To our knowledge, the state shown in Fig. 14 has not been observed experimentally. Therefore, this state is considered to be a metastable state. If we take thermal noise into account, the state will fall into the state shown in Fig. 11(b) and Fig. 12(b).

\section{CONCLUSION}

We derived a set of equations that describe the shape deformation and phase separation dynamics of a twocomponent membrane whose bending moduli depends on the local composition of the lipids. Using the timeevolution equations we demonstrated the effects of (i) thermal noise, (ii) hydrodynamic flow induced by the line tension of the domain boundary and (iii) compositiondependent bending rigidity on the coarsening dynamics of the phase-separated patterns for shape-fixed vesicles (spherical for (i) and (ii), and a tubular for (iii)) in Sec.III A, IIIB and III C, respectively. Regarding to 
the effects of thermal noise, the dynamic exponent $z$ in $N_{\mathrm{DB}} \sim t^{-z}$ of phase separation in $\phi_{\mathrm{o}}=0.3$ is found to be $1 / 3$ with no thermal noise and $1 / 5<z<1 / 4$ with thermal noise $(\zeta>0.1)$. The main coarsening mechanism in the late stage of the system under no thermal noise is the evaporation-condensation process. In the systems with thermal noise $(\zeta>0.1)$, we observed Brownian migrations and domain coalescence in addition to the evaporation condensation process. The decrease of the dynamical exponent $z$ in the systems of $\zeta>0.1$ seems to originate from a significant decrease in the effective line tension, as seen from the large fluctuation of the interface line. We also found that the hydrodynamic effect can accelerate the coarsening in a bicontinuous phase separation of $\phi_{\mathrm{o}}=0$. In the critical quenching $\left(\phi_{\mathrm{o}}=0\right)$, the dynamical exponent of the domain coarsening $z$ seems to be $2 / 3$ in the beginning of the late stage $\tilde{t}>70$, where the average magnitude of the hydrodynamic current is significantly enhanced, and $z$ is close to $1 / 2$ [35] in the time region $70<\tilde{t}<200$. The dynamical exponent finally changes to $1 / 3$ due to the intrinsic finite-size effect of the finite-size vesicle. We also investigated the effect of the composition-dependent bending rigidity on the phase separation dynamics in a shape-fixed tubular vesicle. The coupling of local composition and curvature through $\kappa(\phi)$ was found to induce the formation of ax- isymmetric band-like domains. Finally, using an oblate biconcave shape and a prolate dumbbell shape as the two types of initial shapes prepared by applying an osmotic pressure difference according to the same procedure in the experiment done by Yanagisawa et al[23, 24], we also investigated the dynamics of shape deformation and phase separation of two-component vesicles with a composition-dependent bending rigidity. We found that, after quenching the systems to induce phase separations, almost all of the states that were in biconcave and prolate dumbbell shapes change into states composed of two almost-flat domains rich in type-A lipids with a higher bending rigidity and a cylindrical domain rich in the type-B lipids. This result is in good agreement with the experimentally observed behavior known as shape convergence.

\section{A. Acknowledgments}

This work was supported by the Grant-in-Aid for Scientific Research on Priority Area "Soft Matter Physics" from the Ministry of Education, Culture, Sports, Science and Technology of Japan.
[1] Canham P B 1970 J. Theor. Biol. 2661

[2] Helfrich W 1973 Z. Naturforsch. 28c 693

[3] Lipowsky R $1992 J$ de Physique 2 1825-40

[4] Jülicher F and Lipowsky R 1993 Phys. Rev. Lett. 70 2964-67

[5] Jülicher F and Lipowsky R 1996 Phys. Rev. E 53 2670-83

[6] Lipowsky R and Dimova R 2003 J. Phys. Condens. Matter $15 \mathrm{~S} 31-45$

[7] Gutlederer E, Gruhn T and Lipowsky R 2009 Soft Matter, Soft Matter 5 3303-11

[8] Campelo F, Allain J-M and Amar M B 2007 Eur. Phys. Lett. 77 38006-p5

[9] Komura S, Shimokawa N and Andelman D 2006 Langmuir 22 6771-74

[10] Kawakatsu T, Andelman D, Kawasaki K, and Taniguchi T 1993 J. Phys. II (France) 3 971-997

[11] Taniguchi T, Kawasaki K, Andelman D and Kawakatsu T 1994 J. Phys. II (France) 4 1333-1362

[12] Taniguchi T 1996 Phys. Rev. Lett., 76, 4444-7

[13] Taniguchi T 1997 Progr. Colloid Polym. Sci. 106 274-6

[14] Kumar P B S and Rao M 1998 Phys. Rev. Lett. 80 248992

[15] Kumar P B S, Gompper G and Lipowsky R 2001 Phys. Rev. Lett. 86 3911-14

[16] Laradji M and P B S Kumar 2004 Phys. Rev. Lett. 93 198105

[17] Laradji M and P B S Kumar 2005 J. Chem. Phys. 123 224902

[18] Baumgart T, Hess S T and Webb W W 2003 Nature 425 821-824

[19] Veatch S L and Keller S L 2005 Phys. Rev. Lett. 94
148101

[20] Tian A, Johnson C, Wang W and Baumgart T 2007 Phys. Rev. Lett. 98208102

[21] Honerlamp-Simith A R, Cicuta P, Collins M D, Veatch S L, Nijs M, Schick M and Keller S L 2008 Biophys. J. 95 236-46

[22] Yanagisawa M, Imai M, Masui T, Komura S and Ohta T 2007 Biophys. J. 92 115-25

[23] Yanagisawa M, Imai M and Taniguchi T 2008 Rhys. Rev. Lett. 100148102

[24] Yanagisawa M, Imai M and Taniguchi T 2008 Progress of Theoretical Physics Supplement 175 71-80

[25] Sakuma, Y., M. Imai, M. Yanagisawa, and S. Komura. Eur. Phys. J. E. 25403 (2008).

[26] Semrau S, Idema T, Holtzer L, Schmidt T and Storm C 2008 Phys. Rev. Lett. 100088101

[27] Sakuma Y, Taniguchi T and Imai M 2010 Biophysical Journal, 99 472-479

[28] Parthasarathy R, Yu C and Groves J T 2006 Langmuir 22 5095-99

[29] Rozovsky S, Kaizuka Y and Groves J T 2005 J. Am. Chem. Soc. 127 36-7

[30] Seki K, Komura S and Imai M 2007 J. Phys. Condens. Matter 19072101

[31] Inaura K and Fujitani Y 2008 Journal of the Physical Society of Japan, $\mathbf{7 7} 114603$

[32] Fujitani Y 2009 Journal of the Physical Society of Japan 78084402

[33] Lifshitz E M and Pitaevskii L P, Statistical Physics Part 2, Chapter 9 (Butterworth Heinemann)

[34] Jenkins J T 1977 J. Math. Biology 4 149-69 
[35] Ramachandran S, Komura S and Gompper G 2010 Europhys. Lett. 8956001

[36] Taniguchi T and Onuki A 1997 Phys. Rev. Lett., 77,
4910-13

[37] Ziherl P and Svetina S 2005 Europhys. Lett. 94 690-6 